Article

\title{
Protein Elicitor PeaT1 Efficiently Controlled Barley Yellow Dwarf Virus in Wheat
}

\author{
Lin $\mathrm{Li}^{1,2}{ }^{10}$, Shuangchao Wang ${ }^{1}$, Xiufen Yang ${ }^{1}$, Frederic Francis ${ }^{2, *}$ and Dewen Qiu ${ }^{1, *}$ \\ 1 State Key Laboratory of Plant Diseases and Insect Pests, Institute of Plant Protection, Chinese Academy of \\ Agricultural Sciences, Beijing 100193, China \\ 2 Functional and Evolutionary Entomology, Gembloux Agro-Bio Tech, University of Liège, \\ Gembloux B-5030, Belgium \\ * Correspondence: Frederic.Francis@uliege.be (F.F.); qiudewen@caas.cn (D.Q.); \\ Tel.: +32-8162-2283 (F.F.); +86-10-8210-5929 (D.Q.)
}

Received: 5 August 2019; Accepted: 4 September 2019; Published: 6 September 2019

\begin{abstract}
Barley yellow dwarf virus (BYDV), transmitted by the wheat aphid, generates serious wheat yellow dwarf disease and causes great losses in agriculture. Induced resistance has attracted great attention over recent years as a biological method to control plant pathogens and herbivores. Protein elicitor PeaT1 induces defense response in plants against fungi, viruses, and aphids. In this study, wheat seeds and seedlings were soaked and sprayed with $30 \mu \mathrm{g} / \mathrm{mL}$ PeaT1, respectively. Then seedlings were inoculated with BYDV by viruliferous Schizaphis graminum to detect the control efficiency of PeaT1-induced resistance against BYDV. The control efficiency was over 30\% on the 14th and 21st days after the inoculation access period. Quantitative real time polymerase chain reaction (Q-RT-PCR) tests showed that there was less mRNA from the BYDV coat protein in PeaT1-treated wheat seedlings than in the control group. Electrical penetration graph (EPG) tests showed that virus transmission vector S.graminum took a longer time to find probe and feeding sites on PeaT1-treated wheat seedlings. Additionally, PeaT1-treated wheat seedlings gained higher plant height and more chlorophyll a\&b. These results showed that PeaT1 efficiently controlled BYDV by inhibiting BYDV proliferation, reducing the virus transmission ability of $S$. graminum and alleviating the symptoms of dwarfism and yellow colouring caused by BYDV. This study provided a new integrated way to control BYDV biologically.
\end{abstract}

Keywords: barley yellow dwarf virus; protein elicitor; PeaT1; EPG; virus transmission ability; virus proliferation

\section{Introduction}

Barley yellow dwarf virus (BYDV), Luteoviridae luteovirus, is a virus with worldwide distribution, which is persistently and circulatively transmitted by aphids, and hosted mostly in the phloem of Poaceae plants [1]. BYDV causes serious yellow and dwarf disease in Triticum, with chlorosis leaves, dwarf plants, retarded growth, less tillering, and maldevelopment of roots and spikes, leading to yieldloss. Today, control of BYDV is mainly performed by culturing resistant strains of wheat using crossing breeding, which is time and labor consuming, and chemical control of the virus transmission vector aphid, which makes it easy for aphids to develop pesticide resistance and contaminates the environment [2-4].

Recently, induced resistance has aroused hot discussion as a new biological measure in controlling plant pathogens and herbivores. Induced resistance implies that plants initiate the immune system with an enhanced defense response when they have failed to defend themselves with constitutive defense mechanisms against biotic or abiotic stresses [5]. The defense responses consists of hypersensitive 
response (HR), enhancement of cell wall, accumulation of phytohormones and production of secondary metabolites, leading to toxicity or deterrent against pathogens or herbivores [6,7]. In one study, Bacillus cereus was introduced into tomato plants by seed microbiolization and enhanced tomato plant resistance against Alternaria solani, Phytophthora infestans and Septoria lycopersici [8]. Plant hormones, jasmonic acid, and salicylic acid have been show to induce plant defense responses against pathogens and herbivores $[9,10]$. Some compounds discovered from plant pathogens were also found to be efficient in triggering plant defense responses and enhanced plant resistance, like PeBA1 (Protein elicitor from Bacillus amyloliquefaciens NC6) and AsES (Acremonium strictum Elicitor Subtilisin) [6,11]. It could be concluded that induced resistance could be employed to control plant pathogens and herbivores in agriculture.

Compounds that trigger plant defense response were called elicitors, including pathogen/herbivoreassociated molecular patterns (PAMP/HAMP), effectors and other abiotic stimulus [12,13]. Elicitors are advantageous in biological control with less selective pressure on pathogens and herbivores, and are much more environmentally friendly compared with chemical pesticide. PeaT1 (GenBank No. ABM54184.1) has been isolated from Alternaria tenuissima, triggering defense responses in various plants against fungi, viruses, aphids, salt and drought [14-16], and showing no direct toxicity to plant pathogenic microorganisms and aphids. In this study, we evaluated PeaT1-induced resistance in wheat against BYDV and its effects on the feeding behavior of virus transmission vector Schizaphis graminum, exploring an integrated method to control BYDV.

\section{Material and Method}

\subsection{Materials}

BYDV-GAV and plasmid (pDHB1)-containing coat protein gene were gifted by Professor Liu Yan from the Institute of Plant Protection, Chinese Academy of Agricultural Sciences (IPP, CAAS, Bejing, China). Schizaphis graminum was gifted by Dr. Zhang Yong from IPP, CAAS. Seeds of Triticum aestivum L.Zhongmai 175 were gifted by Dr. Li Faji from Institute of Crop Sciences, CAAS. System of wheat-BYDV-S. graminum was cultured under $23 \pm 1{ }^{\circ} \mathrm{C}$ and $16 \mathrm{~h}$ light. Protein elicitor PeaT1 was expressed by Escherichia coli-pET28-PeaT1-His [7].

\subsection{Bioassay}

Wheat seeds were soaked in PeaT1 $(30 \mu \mathrm{g} / \mathrm{mL})$ for $24 \mathrm{~h}$ under temperature conditions of $23 \pm 1{ }^{\circ} \mathrm{C}$ and washed by water before being planted (six seeds per pot). Seven days later, seedlings were sprayed with PeaT1 (30 $\mu \mathrm{g} / \mathrm{mL}$, and treated identically every 7 days). One day after seedlings were sprayed with PeaT1 solution, 15-day-old viruliferous apterous $S$. graminum were introduced onto the wheat seedlings (around 4-6 aphids per plant) for 2days (that is 2 days inoculation access period; IAP) and eliminated by dimethyl dichloroviny phosphate (DDVP; $1 \mathrm{mg} / \mathrm{mL}$ ). In the control group, seeds and seedlings were treated with water following the same protocol as PeaT1 treatment. Then the severity of disease was recorded, disease index and control efficiency were calculated on the 14th and 21st days after 2 days IAP. The 2nd and 3rd wheat leaves were chosen as the target of investigation. Severity of disease leaves were divided into 11 grades from 0 to 10 according to chlorosis level (Table 1). The test was repeated three times with six replicates for each treatment.

Disease index $=100 \times \sum($ disease grade $\times$ number of leave $) /($ total number of leave $\times 10), 10$ is the highest disease grade.

Control efficiency $=(1-$ disease index of PeaT1 treatment/disease index of control $) \times 100 \%$. 
Table 1. Grade of disease leave of barley yellow dwarf virus (BYDV)-infected wheat indoor.

\begin{tabular}{cc}
\hline Disease Grade & Grading Standard \\
\hline 0 & Health leaf without chlorosis \\
1 & Top end of leaf is chlorosis \\
2 & The margin of $1-2 \mathrm{~cm}$ top of leaf is light yellow \\
3 & $1-2 \mathrm{~cm}$ top of leaf is yellow \\
4 & $1 / 4$ of leaf is yellow, other part is light chlorosis \\
5 & $1 / 3$ of leaf is yellow, other part is light chlorosis \\
6 & Top end of leaf is dried up, $1 / 2$ of leaf is yellow, other part is light chlorosis \\
7 & Top end of leaf is dried up, over $1 / 2$ of leaf is yellow, other part is light chlorosis \\
8 & Top end of leaf is dried up, whole leaf is chlorosis or yellow nonuniformly \\
9 & Top end of leaf is dried up, whole leaf is yellow \\
10 & whole leaf is yellow or dried up
\end{tabular}

\subsection{Determination of Wheat Height and Chlorophyll Content}

Wheat seeds and seedlings were treated as described above. BYDV inoculation followed the same procedures as above. Wheat height was measured at 14th and 21th day after IAP. At the same time, wheat leaves were collected for determination of chlorophyll a\&b [17]. Each test was repeated three times with 6 replicates.

The content of chlorophyll $\mathrm{a} \& \mathrm{~b}$ was determined using the following equation: $(\mu \mathrm{g} / \mathrm{g} \times$ fresh weight $)=\left(24.93 \times \mathrm{A}_{665}+1.44 \times \mathrm{A}_{652}\right) /$ fresh weight of leaf

\subsection{Quantity Determination of BYDV Coat Protein mRNA by Quantitative Real Time Polymerase Chain Reaction $(Q-R T-P C R)$}

Wheat seeds and seedlings were treated identically and then inoculated with BYDV as described above. On the 3rd, 6th, 9th, 12th and 15th days after IAP wheat leaves were collected for mRNA extraction, cDNA synthesis and absolute quantification of BYDV coat protein gene (TransGen kits, Beijing, China). Primers for the BYDV coat protein gene were: $5^{\prime}$-ATGAATTCAGTAGGCCGTAGA-3' and 5'-CTATTTGGGAGTCATGTTGGC-3' [18]. The standard curve for plasmids with the BYDV coat protein gene was drawn according to the method discussed by Li et al. [19]. The determination was repeated twice with three biological replicates each time.

Increased rates of BYDV coat protein mRNA (\%) were determined using the following equation: (quantity of BYDV of current time point - quantity of BYDV of previous time point)/ quantity of BYDV of previous time point $\times 100$.

\subsection{Detection of Apterous Viruliferous Schizaphis graminum Feeding Behavior}

Wheat seeds and seedlings were treated identically as described above. Electrical penetration graph (EPG; GIGA-8d) tests were conducted 1 day after seedlings spray. Fifteen-day-old viruliferous apterous S. graminum were starved for 1 hour before testing [20]. All of the tests were performed at the same time, from $10 \mathrm{am}$ to $4 \mathrm{pm}$ every day. Stylet ${ }^{+} \mathrm{a} \& \mathrm{~b}$ were used to record and distinguish the waveforms for aphid feeding behavior [21]. Excel workbook was used to analyze the EPG data [22].

\subsection{Data Analysis}

All data were analyzed using SPSS 18.0 (International Business Machines Corporation, Armonk, NY, USA) with the Student's $t$ test and least significant difference (LSD).

\section{Results}

\subsection{Control Efficiency of PeaT1 on BYDV Indoor}

PeaT1-induced resistance performed with good control efficiency against BYDV. The disease index in the PeaT1 treatment group was significantly lower than that of the control group, indicating that 
PeaT1 performed well in terms of its inhibition effect on BYDV. The control efficiency was over $30 \%$ on the 14th and 21st days after IAP (Table 2).

Table 2. Control efficiency of PeaT1 on BYDV indoor.

\begin{tabular}{cccc}
\hline \multirow{2}{*}{ Time after IAP (Day) } & \multicolumn{2}{c}{ Disease Index } & \multirow{2}{*}{ Control Efficiency (\%) } \\
\cline { 2 - 3 } & Control & PeaT1 Treatment & \\
\hline 14 & $27.47 \pm 2.22$ & $17.92 \pm 1.44^{* *}$ & $35.34 \pm 2.20$ \\
21 & $38.93 \pm 0.70$ & $24.58 \pm 3.45^{* *}$ & $36.77 \pm 9.59$ \\
\hline
\end{tabular}

Note: IAP, inoculation access period. Data are shown as mean \pm standard deviation (SD). Two asterisks show significant difference between control and PeaT1 treatment $(p<0.01)$ in same investigation time.

\subsection{Effect of PeaT1 on BYDV Coat Protein mRNA}

According to Figure 1, the amounts of BYDV coat protein mRNA in PeaT1-treated wheat seedlings were significantly less than that of the control group on the 3rd, 12th and 15th days after IAP, with a smaller increase rate on the 12th and 15th days. These results suggested that PeaT1 significantly inhibited the proliferation of BYDV.

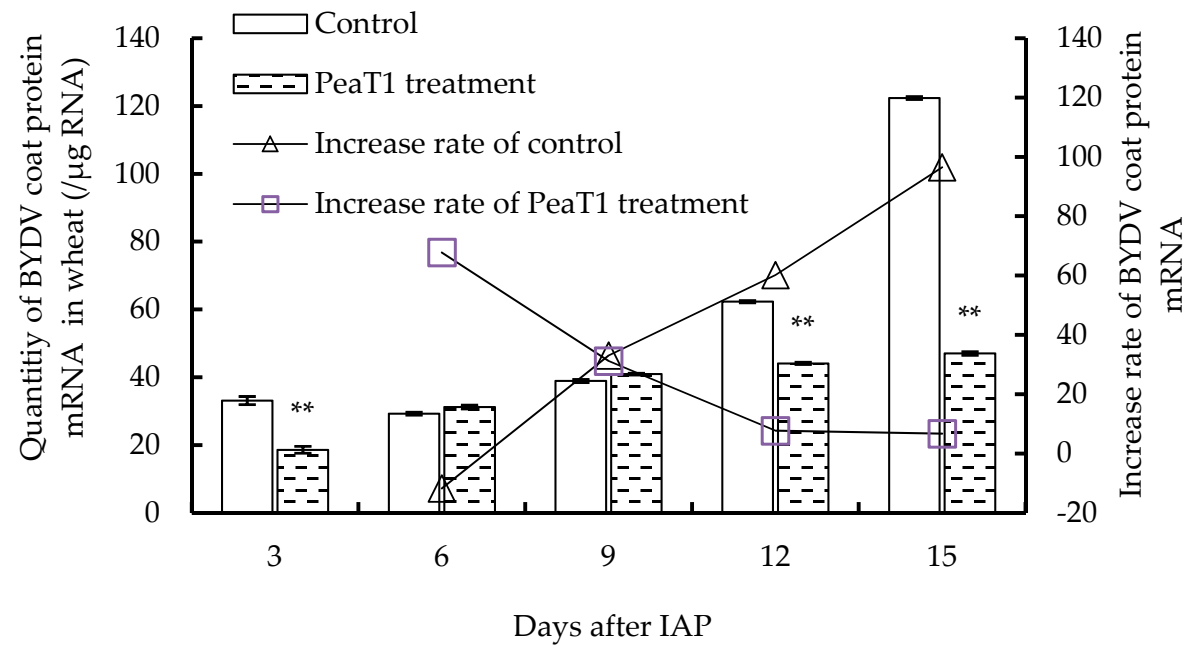

Figure 1. Effect of PeaT1 on the quantity of barley yellow dwarf virus (BYDV) coat protein mRNA in wheat. Note: two asterisks stand for the significant difference in BYDV quantity between control and PeaT1 treatment, $p<0.01$.

\subsection{Effect of PeaT1 on Transmission Vector Schizaphis graminum Feeding Behavior}

According to Table 3, the time to the first probe from the start of EPG was longer in PeaT1-treated wheat seedlings, suggesting a longer time for S. graminum to find a probe site on the leaf surface. The duration of the nonprobe period before the 1st E1 (saliva secretion during phloem feeding activities), time from 1st probe to 1st E1, and time from the start of EPG to the 1st E1 were longer in PeaT1-treated wheat seedlings than in those of the control group, indicating that S. graminum took a longer time to find the feeding site in the phloem. These results showed that PeaT1 negatively affected feeding behavior, and furthermore, reduced the BYDV transmission ability of S. graminum. 
Table 3. Electrical penetration graph (EPG) parameters of Schizaphisgraminumin $6 \mathrm{~h}$ feeding time.

\begin{tabular}{cccc}
\hline Parameters of EPG & Control $(\boldsymbol{n = 2 2 )}$ & $\begin{array}{c}\text { PeaT1 Treatment } \\
(\boldsymbol{n}=\mathbf{2 2})\end{array}$ & $p$ Value \\
\hline Time to 1st probe from start of EPG (min) & $4.20 \pm 0.64$ & $7.25 \pm 0.87^{*}$ & 0.04 \\
Duration of nonprobe period before the 1st E1 (min) & $18.11 \pm 2.22$ & $27.69 \pm 3.94^{*}$ & 0.05 \\
Time from 1st probe to 1st E1 (h) & $1.80 \pm 0.14$ & $2.50 \pm 0.28^{*}$ & 0.04 \\
Time from start of EPG to 1st E1 (h) & $1.86 \pm 0.14$ & $2.53 \pm 0.28^{*}$ & 0.05 \\
Mean duration of E1 (min) & $2.28 \pm 0.28$ & $2.90 \pm 0.49$ & 0.32 \\
\hline
\end{tabular}

Note: Data are shown as mean \pm SD. $n$ was the valid record number of each treatment. One asterisk shows difference in same parameter between control and PeaT1 treatment, $p<0.05$.

\subsection{PeaT1 Weakened the Symptom of Wheat Caused by BYDV}

BYDV causes yellow leaves and plant dwarfism in wheat. In this study, we found PeaT1 alleviated the symptoms in BYDV-infected wheat (Figure 2). Both the chlorophyll content and plant height were higher in PeaT1-treated wheat seedlings than in the controls (Table 4).

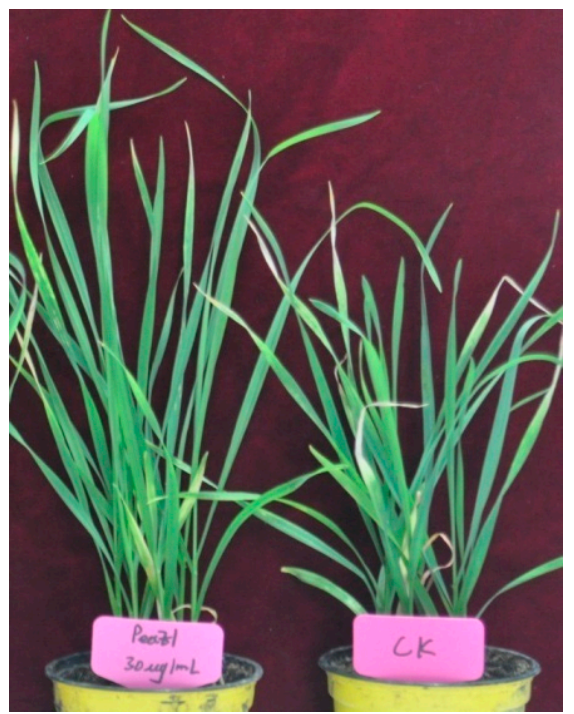

Figure 2. PeaT1 alleviated symptoms of dwarfism and yellow leaves in wheat caused by BYDV.

Table 4. Content of chlorophyll and wheat height in control and PeaT1-treated wheat seedlings.

\begin{tabular}{ccccc}
\hline \multirow{2}{*}{$\begin{array}{c}\text { Time after IAP } \\
\text { (Day) }\end{array}$} & \multicolumn{2}{c}{$\begin{array}{c}\text { Content of Chlorophyll } \mathbf{a} \& \mathbf{b} \\
(\mu \mathrm{g} / \mathbf{g} \times \mathbf{F W})\end{array}$} & \multicolumn{2}{c}{$\begin{array}{c}\text { Wheat Height } \\
(\mathbf{c m})\end{array}$} \\
\cline { 2 - 5 } & Control & PeaT1 Treatment & Control & PeaT1 Treatment \\
\hline 14 & $144.29 \pm 4.58$ & $194.23 \pm 7.05^{* *}$ & $26.88 \pm 1.3$ & $33.35 \pm 1.56^{* *}$ \\
21 & $106.06 \pm 7.56$ & $172.64 \pm 5.29^{* *}$ & $27.59 \pm 1.82$ & $33.41 \pm 2.54^{* *}$ \\
\hline
\end{tabular}

Note: Data are shown as mean \pm SD. IAP, inoculation access period. Two asterisks show significant difference in content of chlorophyll or wheat height in same investigation time between control and PeaT1 treatment, $p<0.01$. FW stands for fresh weight of wheat leaves.

\section{Discussion}

In this study, we found that PeaT1 could control the BYDV at a rate of over $30 \%$ on the 7 th and 14th day after IAP. Q-RT-PCR results showed that the amount and increase rate of BYDV coat protein mRNA were lower in PeaT1-treated wheat seedlings. Induced resistance could enhance plant defense against pathogen viruses [23,24]. PeaT1 has been found to induce salicylic acid pathway-dependent systemic acquired resistance in tobacco and rice, elevating the amount of defense-related compounds, such as lignin, abscisic acid, malondialdehyde and proline $[7,25]$. PeaT1 was supposed to trigger plant 
defense responses, induce accumulation of defense-related compounds in wheat, enhancing the wheat resistance and leading to significant control efficiency for BYDV.

BYDV was mainly located in the plant phloem, including sieve elements, companion cells and parenchymal cells [26]. Generally, BYDV was acquired and inoculated by wheat aphids during the phloem feeding process. The virus entered into aphids by sap sucking (E2 wave in EPG) and into plants by saliva secretion (E1 wave in EPG) [27]. The duration of E1 was positively related with the virus transmission ability of aphids. However, in this study, the duration of E1 wasn't affected by PeaT1. According to our results, S. graminum took a longer time to find the probe site and feeding site, indicating PeaT1-treated wheat seedlings inhibited feeding behavior of S. graminum, which was also proved in S. avenae with shorter total probing time and longer duration of non-probe time before the first E1 [15]. Givovich and Niemeyer [28] found that BYDV transmission ability decreased when aphid feeding behavior was inhibited by a resistant strain of wheat, which was shown by the longer time to phloem feeding from the start of EPG. As a result, PeaT1-treated wheat seedlings inhibited feeding behavior of $S$. graminum, and furthermore inhibited the BYDV transmission ability.

BYDV causes a hazard for the wheat plant by destroying the chloroplast and decreasing chlorophyll content [29]. In this study, we found that PeaT1 increased the chlorophyll content of BYDV-infected wheat, which showed that PeaT1 alleviated the effect of PeaT1 on chloroplast. In addition, PeaT1-treated wheat seedlings were taller than control wheat seedlings, suggesting that PeaT1 weakened the dwarfism symptom caused by BYDV. As a result, PeaT1 was supposed to reduce the effects of BYDV on wheat.

Above all, PeaT1 controlled BYDV efficiently by inhibiting the proliferation of BYDV in wheat and decreasing the efficiency of virus transmission by S. graminum. Additionally, PeaT1 alleviated symptoms caused by BYDV in wheat. PeaT1 has been developed into a commercial pesticide (Atailing) and applied to wheat in the field to control wheat sheath blight, powdery mildew and leaf rust, promote wheat growth, and increase yield [30]. Protein elicitor is easy to degrade and less stressful for aphids to develop pesticide resistance, which meet the requirement of food and environmental safety. In conclusion, PeaT1 is a prospective biological agent to be applied in BYDV control in the field. However, wheat growth, aphids, disease development and the abiotic environment are quite different between controlled conditions and in the field. Therefore, the mode of application in the field remains open to further study.

Author Contributions: Conceptualization, X.Y. and D.Q.; Investigation, L.L.; Data Curation, S.W.; Writing-Original Draft Preparation, L.L.; Writing—Review \& Editing, X.Y. and S.W.; Supervision, F.F. and D.Q.; Funding Acquisition, D.Q.

Funding: This work was supported by National Key R\&D Program of China (2017YFD0200900 and 2018YFD0201500) and international cooperation project between China and Belgium (2014DFG32270).

Conflicts of Interest: The authors declare no conflict of interest.

\section{References}

1. Domier, L.L.; D’Arcy, C.J. Luteoviruses. In Encyclopedia of Virology, 3rd ed.; Mahy, B.W.J., Van Regenmortel, M.H.V., Eds.; Academic Press: Oxford, UK, 2008; pp. 231-238.

2. Harris, J. Chemical Pesticide Markets, Health Risks and Residues; CABI Publishing: Wallingford, UK, 2000.

3. MacKenzie, T.D.B.; Arju, I.; Poirier, R.; Singh, M. A genetic survey of pyrethroid insecticide resistance in aphids in New Brunswick, Canada, with particular emphasis on aphids as vectors of potato virus $\mathrm{Y}$. J. Econ. Entomol. 2018, 111, 1361-1368. [CrossRef]

4. Pascher, K.; Gollmann, G. Ecological risk assessment of transgenic plant releases: An Austrian perspective. Biodivers. Conserv. 1999, 8, 1139-1158. [CrossRef]

5. Mauch-Mani, B.; Baccelli, I.; Luna, E.; Flors, V. Defense priming: An adaptive part of induced resistance. Annu. Rev. Plant Biol. 2017, 68, 485-512. [CrossRef]

6. Hael, C.V.; Perato, S.M.; Arias, M.E.; Martínez Zamora, M.G.; Di, P.P.; Martos, G.G.; Castagnaro, A.P.; Diaz-Ricci, J.C.; Chalfoun, N.R. The elicitor protein AsES induces a SAR response accompanied by systemic microbursts and micro-HRs in Fragaria ananassa. Mol. Plant-Microbe Interact. 2017, 31, 46-60. [CrossRef] 
7. Zhang, W.; Yang, X.; Qiu, D.; Guo, L.; Zeng, H.; Mao, J.; Gao, Q. PeaT1-induced systemic acquired resistance in tobacco follows salicylic acid-dependent pathway. Mol. Biol. Rep. 2011, 38, 2549-2556. [CrossRef]

8. Silva, H.S.A.; Romeiro, R.S.; Carrer Filho, R.; Pereira, J.L.A.; Mizubuti, E.S.G.; Mounteer, A. Induction of systemic resistance by Bacillus cereus against tomato foliar diseases under field conditions. J. Phytopathol. 2004, 152, 371-375. [CrossRef]

9. Kouzai, Y.; Kimura, M.; Watanabe, M.; Kusunoki, K.; Osaka, D.; Suzuki, T.; Matsui, H.; Yamamoto, M.; Ichinose, Y.; Toyoda, K.; et al. Salicylic acid-dependent immunity contributes to resistance against Rhizoctonia solani, a necrotrophic fungal agent of sheath blight, in rice and Brachypodium distachyon. New Phytol. 2018, 217, 771-783. [CrossRef]

10. Sanches, P.A.; Santos, F.; Penaflor, M.; Bento, J.M.S. Direct and indirect resistance of sugarcane to Diatraea saccharalis induced by jasmonic acid. Bull. Entomol. Res. 2017, 107, 828-838. [CrossRef]

11. Wang, N.; Liu, M.; Guo, L.; Yang, X.; Qiu, D. A novel protein elicitor (PeBA1) from Bacillus amyloliquefaciens NC6 induces systemic resistance in tobacco. Int. J. Biol. Sci. 2016, 12, 757-767. [CrossRef]

12. Dodds, P.N.; Rathjen, J.P. Plant immunity: Towards an integrated view of plant-pathogen interactions. Nat. Rev. Genet. 2010, 11, 539-548. [CrossRef]

13. Jones, J.D.G.; Dangl, J.L. The plant immune system. Nature 2006, 444, 323-329. [CrossRef]

14. Kulye, M.; Liu, H.; Zhang, Y.; Zeng, H.; Yang, X.; Qiu, D. Hrip1, a novel protein elicitor from necrotrophic fungus, Alternaria tenuissima, elicits cell death, expression of defence-related genes and systemic acquired resistance in tobacco. Plant Cell Environ. 2012, 35, 2104-2120. [CrossRef]

15. Li, L.; Wang, S.; Yang, X.; Francis, F.; Qiu, D. Protein elicitor PeaT1 enchanced resistance against aphid (Sitobion avenae) in wheat. Pest Manag. Sci. 2019. [CrossRef]

16. Peng, X.C.; Qiu, D.W.; Zeng, H.M.; Guo, L.H.; Yang, X.F.; Liu, Z. Inducible and constitutive expression of an elicitor gene Hrip1 from Alternaria tenuissima enhances stress tolerance in Arabidopsis. Transgenic Res. 2015, 24, 135-145. [CrossRef]

17. Lichtenthaler, H.K. Chlorophylls and carotenoids: Pigments of photosynthetic biomembranes. Methods Enzymol. 1987, 148, 350-382.

18. Wang, Y.; Zhou, K.; Wang, X.; Zhou, G. Studies on the movement of Barley yellow dwarf virus-GAV in oat plant by RT-PCR (in Chinese). Acta Phytopathol. Sin. 2009, 39, 249-253.

19. Li, L.; Zhao, C.; Li, H.; Li, W.; Zhang, L.; Xu, D.; Wang, J.; Li, H. Establishment of the plasmid standard curve generation method for absolute quantification PCR (in Chinese). J. Agric. Biotechnol. 2011, 19, 1157-1162.

20. Zhang, Y.; Fan, J.; Francis, F.; Chen, J. Watery saliva secreted by the grain aphid Sitobion avenae stimulates aphid resistance in wheat. J. Agric. Food Chem. 2017, 65, 8798-8805. [CrossRef]

21. Tjallingii, W.F.; Esch, T.H. Fine structure of aphid stylet routes in plant tissues in correlation with EPG signals. Physiol. Entomol. 1993, 18, 317-328. [CrossRef]

22. Sarria, E.; Cid, M.; Garzo, E.; Fereres, A. Excel workbook for automatic parameter calculation of EPG data. Comput. Electron. Agric. 2009, 67, 35-42. [CrossRef]

23. Ma, Z.; Li, W.; Wang, H.; Zhang, X. Induced resistance to tobacco mosaic virus (TMV) on tobacco by botanical-derived VFB. Acta Phytopathol. Sin. 2010, 40, 419-425.

24. Wang, B.; Wang, S.; Tan, B.; Qiu, D.; Yang, X. Systemic acquired resistance to tobacco mosaic virus (TMV) induced by protein elicitor from Vericillium dahliae (PevD1) and its mechanisms in tobacco. J. Agric. Biotechnol. 2012, 20, 188-195.

25. Shi, F.; Dong, Y.; Zhang, Y.; Yang, X.; Qiu, D. Overexpression of the PeaT1 elicitor gene from Alternaria tenuissima improves drought tolerance in rice plants via interaction with a Myo-inositol oxygenase. Front. Plant Sci. 2017, 8, 970. [CrossRef]

26. Gray, S.; Gildow, F.E. Luteovirus-aphid interactions. Annu. Rev. Phytopathol. 2003, 41, 539-566. [CrossRef]

27. Prado, E.; Tjallingii, W.F. Aphid activities during sieve element punctures. Entomol. Exp. Appl. 1994, 72, 157-165. [CrossRef]

28. Givovich, A.; Niemeyer, H.M. Hydroxamic acids affecting barley yellow dwarf virus transmission by the aphid Rhopalosiphum padi. Entomol. Exp. Appl. 1991, 59, 79-85. [CrossRef] 
29. Rong, W.; Wang, X.; Wang, X.; Massart, S.; Zhang, Z. Molecular and Ultrastructural Mechanisms Underlying Yellow Dwarf Symptom Formation in Wheat after Infection of Barley Yellow Dwarf Virus. Int. J. Mol. Sci. 2018, 19, 1187. [CrossRef]

30. Sheng, S.; Zhou, Q.; Qiu, D.; Yang, X. Effects and mechanism of disease resistance and yield improvement induced by plant immune protein preparation Ataling in wheat. Chin. J. Biol. Control 2017, 33, 213-218.

(C) 2019 by the authors. Licensee MDPI, Basel, Switzerland. This article is an open access article distributed under the terms and conditions of the Creative Commons Attribution (CC BY) license (http://creativecommons.org/licenses/by/4.0/). 